\title{
FUNCTIONAL SIMULATION PLATFORM FOR STUDYING UNDERWATER RADIATION PATTERN
}

\author{
Kanwarpreet Gilhotra and Nitaigour Premchand Mahalik \\ Department of Industrial Technology, Jordan College of Agricultural Sciences and Technology, \\ California State University, Fresno, California, USA
}

Received 2014-07-22; Revised 2014-09-09; Accepted 2014-09-11

\begin{abstract}
For underwater communication, sound signals are used in contrast to RF. Recent development in wireless sensor network for underwater applications such as ocean exploration, marine aquaculture, sea animal tracking and so on have motivated the researchers and developers to understand the properties of sound signal within water in details. This study presents work on design and development of a simulation platform which can study the properties of the sound signal in virtual in-water situation. The platform is called functional, because this is a dedicated platform which only deals with the sound signal. The scope of the development includes software development principles and methods, review of signal-specific scientific principles and integration of the principles and laws into the software system via object-oriented strategy considering existing data structures and testing and validation of the platform with exemplar results. The functional platform also incorporates modulation techniques, the type of technology systems to be used and radiation pattern of the signal under the water. The test results show that that functional platform can work for simulation study of radiation pattern of sound signal in underwater scenario.
\end{abstract}

Keywords: Software Development, Simulation Tool, Underwater Application, Sensor Network

\section{INTRODUCTION}

Recent development in wireless sensor network in underwater applications such as ocean exploration, marine aquaculture, sea animal tracking and so on have motivated the researchers and developers to understand the properties of sound signal within water (Lee et al., 2011). Technology plays an important role in underwater exploration and related applications. For example, the modern agriculture accommodates the applications of sensor networking technology in order to monitor, control and manage the seafood farm operations within water. Sensor network has been applied to land agriculture, military, transportation and so on (Akyildiz et al., 2005). In this study, we have presented work on design and development of a simulation platform which can study the properties of the sound signal in-water situation virtually.
Simulation platform is referred to as virtual environment that provides foundation for system development and be used to carry out design studies including conceptual design and evaluation prior to the real design. Standard software packages such as POYNTING (Fourie and Nitch, 2000), Stateflow and Simulink (Bouzoualegh et al., 2005), so on are available and can be used to study the radiation patterns, but this work considers several factors including the modulation technique.

\section{REVIEW}

Simulation play important role in design, testing, optimization and validation. Simulation software is application programs run as graphic-like platform for the interactive design of systems (Mishra, 2010). Simulation technologies provide opportunity to the developer and Technology, California State University, Fresno, California, USA 
system integrator to model, explore and try out a variety of design strategies that eventually becomes a matured design. This additional attempt within design paradigm, called Optimization Layer (OL) can improve the capability of the final design specification by reducing the time of the traditional design process (Mishra et al., 2011). The virtual design environment can greatly improve reliability and availability and help to shorten the design-to-implementation cycle by enabling the users to correct errors and to identify optimized design parameters and requirements before they reach at the implementation stage. Simulation thus can provide insights into the effects before significant time and cost has been invested and can be of great benefit (Mahalik and Kim, 2008). Utilizing simulation platform for sensor network type systems for signal analysis, in particular, has many advantages and there is great deal of validation and optimization. The significance of this paper is that we have developed a software platform which helps to study the radiation pattern of underwater sensors (transmitters) depending upon the variable conditions of the scientific principles in water and it has broad prospective. We have validated the design through (i) simulation of radiation pattern of underwater transmitter without modulation, (ii) with FHSS modulation, (iii) transmitters in array and (iv) transmission depending on depth, salinity and temperature of water body by using the results already published. In the sequel, we have reviewed (a) Software developed methodology, (b) The Wireless Sensor Network and (c) Scientific principles on acoustic signal.

\section{SOFTWARE DEVELOPMENT}

The primary difficulty in software development arises out of the complexity of the application, the ability of the software interoperable tools and the characteristics of the computer system on which the software is to be developed (Leyton, 2006). It is important to assess objectivity closely to make decision about the tools and methods because of rapid change of hardware technology that affects the performance of the developed platform. So, the design and development of software should consider aspects of modularity, interoperability, scalability and flexibility. As a matter of fact, it is also important to understand the development process and its unique characteristics. Essentially, software development consists of several phases. One commonly accepted model of software life cycle is the Waterfall Model (WFM) as shown in Fig. 1
(Liu, 2013). WFM is a very standard model and mostly used in functional platforms including Apps. The essential phases of WFM approach based software development life cycle are (i) Requirements and Specifications: This phase define required functions and performance characteristics of the software, (ii) Blue print or abstract design: Configuration control and data structures and a testing plan, (iii) Design: Detailed specifications including expected size, defining object linking and embedding, algorithm based on scientific principles, interface data structures, control structures, (iv) Coding: Implementation of the functional tool boxes and linking, (v) System Integration: In-house testing and validation, bottleneck. (vi) Acceptance: Operational testing, documentation and (vii) Maintenance: Refinement, new functions are added and old functions deleted. In this study, we have followed the principle of WFM. Improving the quality of software is another major goal in software engineering. Quality addresses reliability, portability, efficiency and modifiability. Also accuracy, robustness, completeness and consistency are essential attributes (Davis, 1995). Besides, the overall software development principles can be categorized under requirement-engineering, design and coding, testing and management.

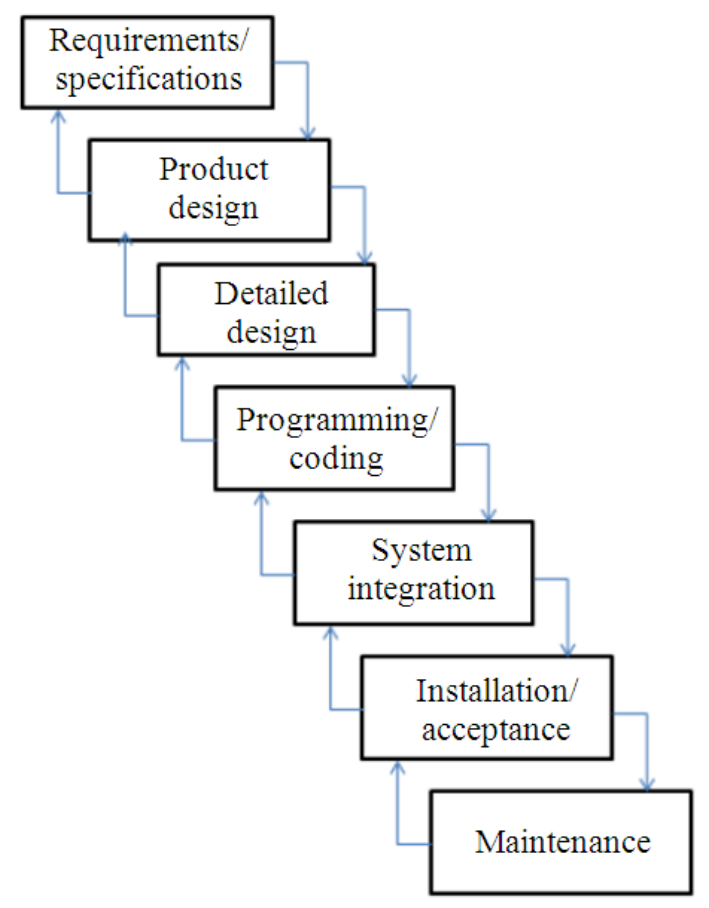

Fig. 1. WFM of software development life-cycle 
Modeling of software is usually considered while developing large scale software. A commonly used modeling paradigm is Unified Modeling Language (UML) (Bock, 2006). There are two main aspects of modeling: Abstraction and rigor. Sometimes, the model includes the user interface. The most important property of modeling is ability to analyze. Other important aspect of software designs is the data handling. Depending upon the requirement, primary data components may be defined within the object executives or classes. However, in real object-oriented scenario, the data are always kept separate from the executives. Several data construct principles are adopted. Some important structures are sequences, selections and iterations which can be combined in an infinite number of ways to form full data structures. In this study two schemes were built in order to deal with handling of data: (i) Draw the input and output data structures and develop interfacings: It provides notation indication to the user at one end and processing tag at the other end to ensure that the data manipulation and handling represent the true requirements for required inputs and outputs and (ii) Tasks for identifying all data components. It builds the data components into data structure with inbuilt fault tolerant capability. Finally, interactive and user friendly interface is developed. The development functional platform is easy to simulate the propagation and radiation patterns under in-water condition. The users can easily design underwater sensor networking system using the GUI. The platform is exceptionally very handy to use as it has controls and toolboxes which are very standard and user friendly.

\section{WSN}

Wireless Sensor Network basically consists of a huge number of sensors. Each of the sensors is very small in respect to its source of energy, memory, power, computational capabilities. WSNs have very unique characteristics: (i) Number of nodes in the wireless sensor network is larger than that of a very typical ad-hoc network, (ii) sensors are basically lowin-cost devices and with severe constraints according to the power, energy source, memory, computation capabilities., (ii) sensors usually are very densely deployed, (iv) probability that sensor will fail is much higher, (v) sensors usually are very stationary rather than moving constantly. Underwater sensor networks are made up of sensors and Autonomous Underwater
Vehicles (AUVs) which are arranged to perform collective monitoring tasks. Some applications of underwater sensor networks are oceanographic data collection, pollution monitoring, offshore exploration, disaster prevention, assisted navigation, tactical surveillance and marine aquaculture and equipped with capability to sense of the physical environment, data processing and to communicate wirelessly to other sensors. Generally, it is assumed that each of the sensors in a network has some certain constraints.

\section{COMMUNICATION TECHNIQUE}

Modulation is the technique defined as the process of varying or changing one of the characteristics of an original waveform that needs transmission with high energy external signal to facilitate transmission for longer distance. Three key changeable parameters are frequency, amplitude and phase. Digital modulation is the process in which we can modulate the base signal with the external digital signal to get the modulating signal. Digital modulation includes Frequency Shift Key (FSK), Amplitude Shift Key (ASK) and Phase Shift Key (PSK) (Nowsheen et al., 2010). The digital modulation can also include Spread Spectrum Communication (SSC). SSC is the techniques which spread the signal over a very broader portion in radio frequency band. Spread spectrum signals use codes which operate many more times the data rate or the information bandwidth. The special spreading-codes are known as pseudo-random codes. In Frequency Hopping Spread Spectrum (FHSS) the transmitting sensor hops between available frequencies in a preassigned sequence in accordance to a specific algorithm (Fig. 2).

Data is transmitted in very short burst over a narrow-band. Then the transmitting sensor is made to get tuned to another different frequency and then transmit again. Thus, the receiving sensor has the capability by which it can hop its frequency many times in a second, over given band width and transmits one frequency for given time and then hops to another frequency and transmits again. In the process of hopping the frequency, carrier frequency is hopped in accordance with the unique sequence so, band width increases. Apparently, the pseudorandom codes are fed to the transmission chain and the receiver chain in same pre-assigned sequence. 

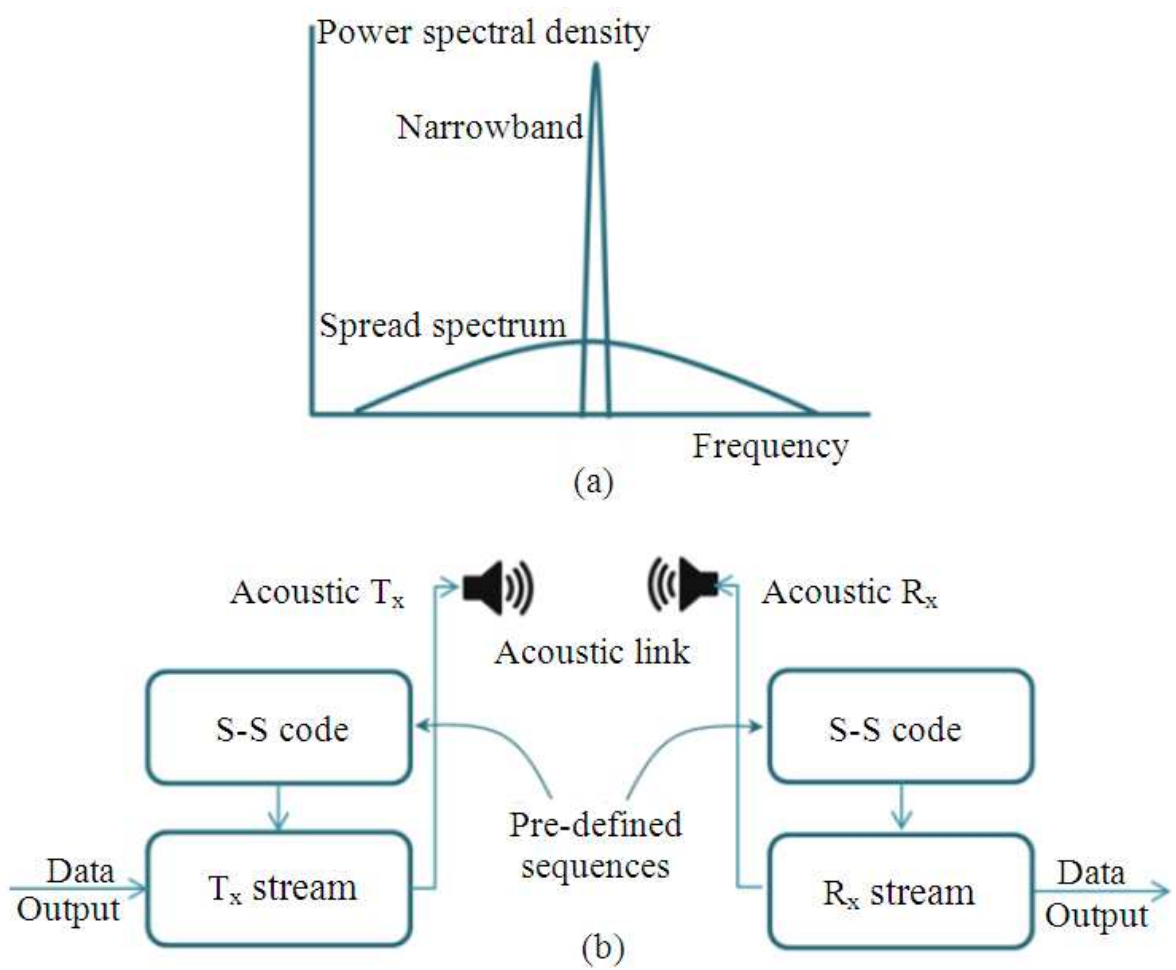

Fig. 2. Principles of spread-spectrum transmission

The advantages of FHSS are (i) resistant to noise, (ii) resistant to jamming, (iii) resistant to interference, (iv) resistant to unauthorized detection, (v) has multiple channels over $2.4 \mathrm{GHz}$ ISM-band. Technical details can be found in (Partan et al., 2006; Jiang, 2008; Pompili et al., 2009). Also, channel modeling plays important role in communication and propagation analysis. The model equations used in this study is presented in Table 1.

\section{PRINCIPLES OF ACOUSTIC SIGNAL}

Acoustic communication works well in water when the communication distance is short. In fact acoustic waves are the only waves which can be used in underwater communications. RF signal from air is converted to acoustic signal and transmitted in water and the process is revered when the transmission occurs in other way round. Acoustic waves are used because optical waves are highly absorbed in water and scattering is significant. On the other hand, acoustic waves have low absorption in underwater environments. Also, radio or RF waves suffer high attenuation demanding higher transmission power and large antennas. Underwater sensors nodes are linked to underwater gateways by wireless acoustic links. Underwater gateways are devices that forward the data from the ocean bottom network to a surface station. Acoustic propagation and radiation patterns in water are expressed using mathematical modeling. The model equations used in this study is presented in Table 2 (Radziejewski, 1990; Yonghun and Kim, 2014; Chen and Pompili, 2014).

\section{RESULTS}

This study presents work on design and development of a functional simulation platform which can study the properties of the sound signal inwater situation virtually. The platform is called functional, because this is a dedicated tool which only deals with the sound related signals and systems. The scope of the development includes software development principles and methods, review of signal-specific scientific principles and integration of the principles and laws into the software system via 
object-oriented strategy and testing and validation of the platform with some exemplar signals and data. The functional platform incorporates modulation techniques, the type of technology systems to be used and radiation pattern of the signal under the water. The test results show that the functional platform can work for simulation study of radiation pattern of sound signals and exploit the channel conditions in underwater scenarios.

In the study, we used Windows based laptop PC and a simple GUI based development tool such as MS Visual Basic 2010 Express version.

Table 1. MAC layer implementation of FHSS (Pompili et al., 2009)

\begin{tabular}{|c|c|}
\hline Model equations & Description \\
\hline$T_{o} \geq \frac{c_{e h}}{R} \cdot L_{e h}+\frac{c_{i j}}{R} \cdot L_{D}$ & MAC protocol description: \\
\hline$+\frac{2 d_{i j}}{\dot{q}}+\frac{c_{a c k}}{R} \cdot L_{a c k}$ & $T_{0}:$ Time out \\
\hline & $\begin{array}{l}c_{e h}: \text { extended header } \\
L_{e h}: \text { extended header size } \\
c_{i j}: \text { code length } \\
L_{D}: \text { actual data packet size } \\
d_{i j}: \text { distance between nodes } i \text { and } j \\
c_{a c k}: \text { acknowledgement code } \\
L_{a c k}: \text { acknowledge packet } \\
R: \text { chip rate and } q: \text { distance }\end{array}$ \\
\hline$c_{i j}^{N_{i j}^{T}}=\min \left[c_{i j}^{N_{i j}^{T-1}} \cdot 2^{\beta}, c_{\max }\right]$ & Successful decoding of packets: \\
\hline$N_{i j}^{T} \in\left[1, N_{\max }^{T}\right]$ & $\begin{array}{l}N_{i j}^{T} \text { : transmission number } \\
\beta \text { : Aggressiveness }\end{array}$ \\
\hline$\frac{N^{0}+I_{j}}{\frac{P_{i j}}{T L_{i j}}}$ & Transmission constraints: \\
\hline$\leq w_{i j} \cdot \Phi\left(B E R_{j}\right)$ & $N^{0}:$ Average noise power \\
\hline$\frac{N^{0}+I_{k}+\frac{P_{i j}}{T L_{i k}}}{S_{k}}$ & $I_{j}:$ User access interference at $i$ \\
\hline$\leq w_{t_{k}} \cdot \Phi\left(B E R_{k}\right)$ & $I_{k}:$ User access interference at $j$ \\
\hline$\forall k \in \mathbf{K}_{i}$ & $\begin{array}{l}P_{i j}: \text { Power by node } i \text { to node } j \\
T L_{i j}: \text { Tx loss from } i \text { to } j \\
T L_{i k}: \text { Tx loss from } j \text { to } j \\
w_{i j}: \text { Bandwidth factor from } i \text { to } k \\
\Phi: \text { Monotonically function } \\
\text { BER: Bit error rate; } \\
w_{i k}: \text { From } t_{k} \text { to } k \\
K_{i}: \text { Power loss values } \\
S_{k}: \text { Received power }\end{array}$ \\
\hline
\end{tabular}

Table 2. Scientific principles of acoustic signals (radiation and propagation) (Radziejewski, 1990)

\begin{tabular}{|c|c|}
\hline Model equations & Description \\
\hline$\frac{\partial^{2} p}{\partial t^{2}}=$ & Propagation: \\
\hline$v^{2}\left(\frac{\partial^{2} p}{\partial x^{2}}+\frac{\partial^{2} p}{\partial y^{2}}+\frac{\partial^{2} p}{\partial z^{2}}\right)$ & (i) Non-homogeneous medium. \\
\hline & $\begin{array}{l}\text { (ii) Cartesian coordinates: } \\
p \text { : Pressure; } \\
v \text { : Velocity of sound. }\end{array}$ \\
\hline$\alpha_{s l}=10 \log \left[1-0.139(f h)^{\frac{3}{2}}\right]$ & $\begin{array}{l}\text { Surface reflection: } \\
\boldsymbol{\alpha}_{s l} \text { : function of the wave; } \\
\mathrm{h} \text { : height of the wave in meters; } \\
\text { f: frequency of waves in } \mathrm{kHz}\end{array}$ \\
\hline$t_{p}=\frac{l}{v}$ & $\begin{array}{l}\text { Propagation time: } \\
l: \text { - path width; } \\
m \text { and } v \text { is the sound speed in } \mathrm{m} / \mathrm{s} \text {. }\end{array}$ \\
\hline$L=20 \log l+n_{s b} \alpha_{s l}$ & Loss: \\
\hline$n_{b b} \alpha_{b l}+l \alpha$ & $\begin{array}{l}n_{s b}: \text { No. of surface bounces; } \\
n_{b b}: \text { No. of bottom bounces; } \\
\alpha_{s l}: \text { Surface reflection loss in } \mathrm{dB} ; \\
\alpha_{b l}: \text { Bottom reflection loss in } \mathrm{dB} ; \\
\alpha: \text { Absorption loss in } \mathrm{dB} / \mathrm{m}\end{array}$ \\
\hline$A_{m}=10^{-\frac{\text { loss }}{20}} *(-1)^{n} s l$ & Attenuation: \\
\hline$t_{d}=t_{p}-t_{0}$ & Time delay: \\
\hline
\end{tabular}

$t_{0}$ : propagation delay of the first signal to arrive at $R x$

$\theta=2 \pi f_{c} t_{d} \quad$ Phase angle:

$S=1+\left[\frac{t_{d}}{T}\right] \quad f_{c}$ : carrier frequency

$d_{t}-t_{d}-(S-1) * T \quad$ Baseband signal:

$T$ : is the length of the symbol period

$r I_{1}=A_{m} \frac{\left(T-d_{T}\right)}{T} \cos \theta \quad$ Phase delay:

$r Q_{1}=-A_{m} \frac{\left(T-d_{T}\right)}{T} \sin \theta \quad I$ and $Q$ components:

$r I_{2}=A_{m} \frac{\left(d_{T}\right)}{T} \cos \theta$

$r Q_{2}=-A \frac{d_{T}}{T} \sin \theta$

$e=r I^{2}+r Q^{2}$

$\sigma=\frac{\sqrt{x^{2}+y^{2}} \operatorname{Sin}\left(\phi+\arctan \frac{x}{y}\right)}{v}$

$r I_{1}$ : phase component of the signal received

$r Q_{1}$ : quadrature components

$r I_{2}$ : phase component of the signal received; $r Q_{2}$ : quadrature components of the signal received during sym-bol period $\mathrm{s}+1$

\section{Delayed signal:}

$x$ and $y$ : Coordinates of receiver hydrophone; $\sigma$. Steering angle

$$
\begin{aligned}
& r I=r I \cos \left(2 \pi f_{c} \sigma\right) \\
& -r Q \sin \left(2 \pi f_{c} \sigma\right) \\
& r Q=r I \sin \left(2 \pi f_{c} \sigma\right) \\
& +r Q \cos \left(2 \pi f_{c} \sigma\right)
\end{aligned}
$$$$
\text { Phase-shifted signal }
$$ 
For testing and verification we employed exact data and results that were published in (Radziejewski, 1990). The published data and results were considered in order to justify that our functional platform is in accordance with the theory and available information. Bearing in mind that the goal of this research was to develop the functional platform, we focused on how to realize the scientific principles and embed them in a small-scale software platform. We have plotted results around several parameters. Figure 3 shows the screen shot of the basic outlook of the simulation software when opened. It displays various shortcuts created to run the simulation under different conditions. In the options menu line color can be selected and the logarithmic scale can be changed accordingly depending upon the situations. Option to download the simulation image in various forms is also available. Play tab is displayed in green to run the software. For the simulated results shown in Fig. 6-8, the parameters that were taken is listed in Table 3.
Figure 4 shows the screenshot of the software in active mode. Graph panel opens up where the simulation run can be displayed. Figure 5 show the screen shot of an illustration where the software was running to simulate radiation pattern of sensor without modulation.

Table 3.Parameters chosen for testing the functional

\begin{tabular}{llll}
\multicolumn{2}{c}{ simulation platform } & & \\
& Value & & \\
\cline { 2 - 4 } Parameters & Sim1 & Sim2 & Sim3 \\
\hline Depth of water & $10 \mathrm{~m}$ & $10 \mathrm{~m}$ & $10 \mathrm{~m}$ \\
Transmitter depth & $5 \mathrm{~m}$ & $5 \mathrm{~m}$ & $4 \mathrm{~m}$ \\
Horizontal distance & variable & $5 \mathrm{~m}$ & variable \\
Bottom loss & $10 \mathrm{~dB}$ & $10 \mathrm{~dB}$ & $10 \mathrm{~dB}$ \\
Carrier frequency & $24 \mathrm{kHz}$ & $24 \mathrm{kHz}$ & $24 \mathrm{kHz}$ \\
Propagation speed & $1477 / \mathrm{m}$ & $1489 / \mathrm{m}$ & $1492 / \mathrm{m}$ \\
Receiver depth & $5 \mathrm{~m}$ & variable & $4 \mathrm{~m}$ \\
Surface loss & $3 \mathrm{~dB}$ & $3 \mathrm{~dB}$ & $3 \mathrm{~dB}$ \\
Absorption loss & $3 \mathrm{~dB} / \mathrm{km}$ & $3 \mathrm{~dB} / \mathrm{km}$ & $3 \mathrm{~dB} / \mathrm{km}$ \\
Symbol rate & $1 \mathrm{kHz}$ & $1 \mathrm{kHz}$ & $1 \mathrm{kHz}$ \\
Sim1: Energy Vs. distance & & & \\
Sim2: Energy Vs. receiver depth & & \\
Sim3: Energy with receiver array & & & \\
\hline
\end{tabular}

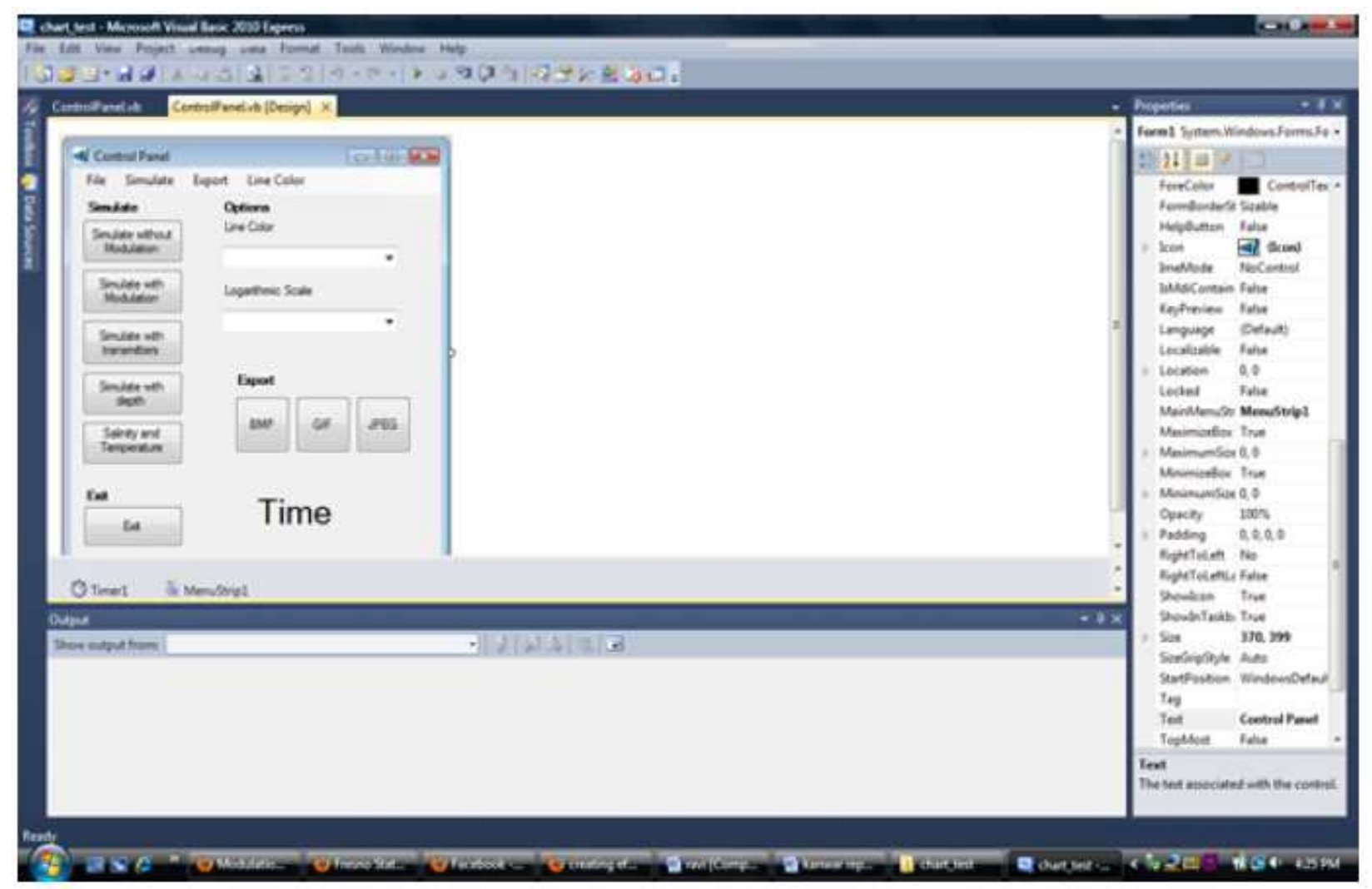

Fig. 3. Screenshot of simulation software (inactive mode) 
K. Gilhotra and N.P. Mahalik / American Journal of Engineering and Applied Sciences 7 (3): 327-337, 2014

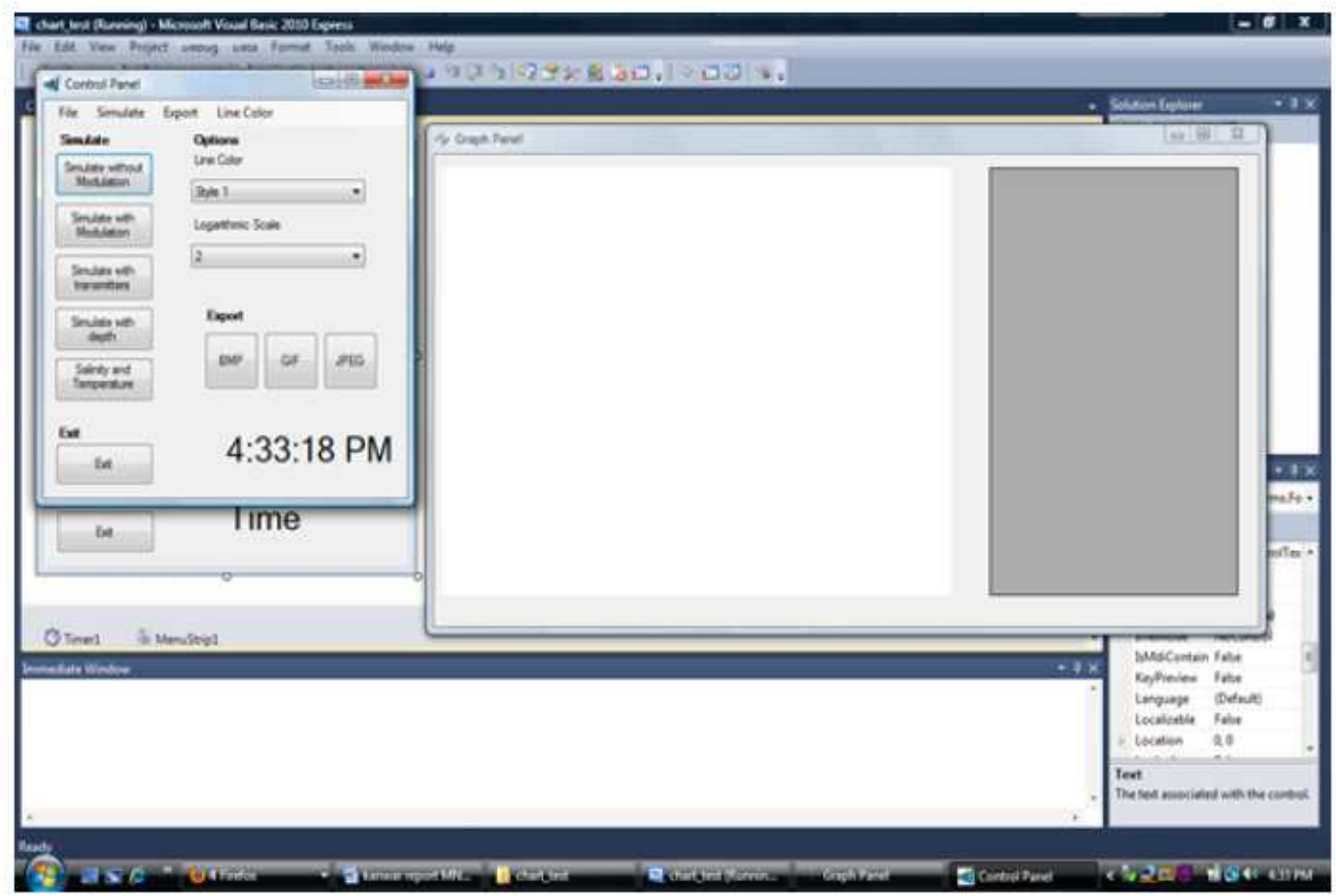

Fig. 4. Screenshot of the software in run mode waiting to receive user defined data

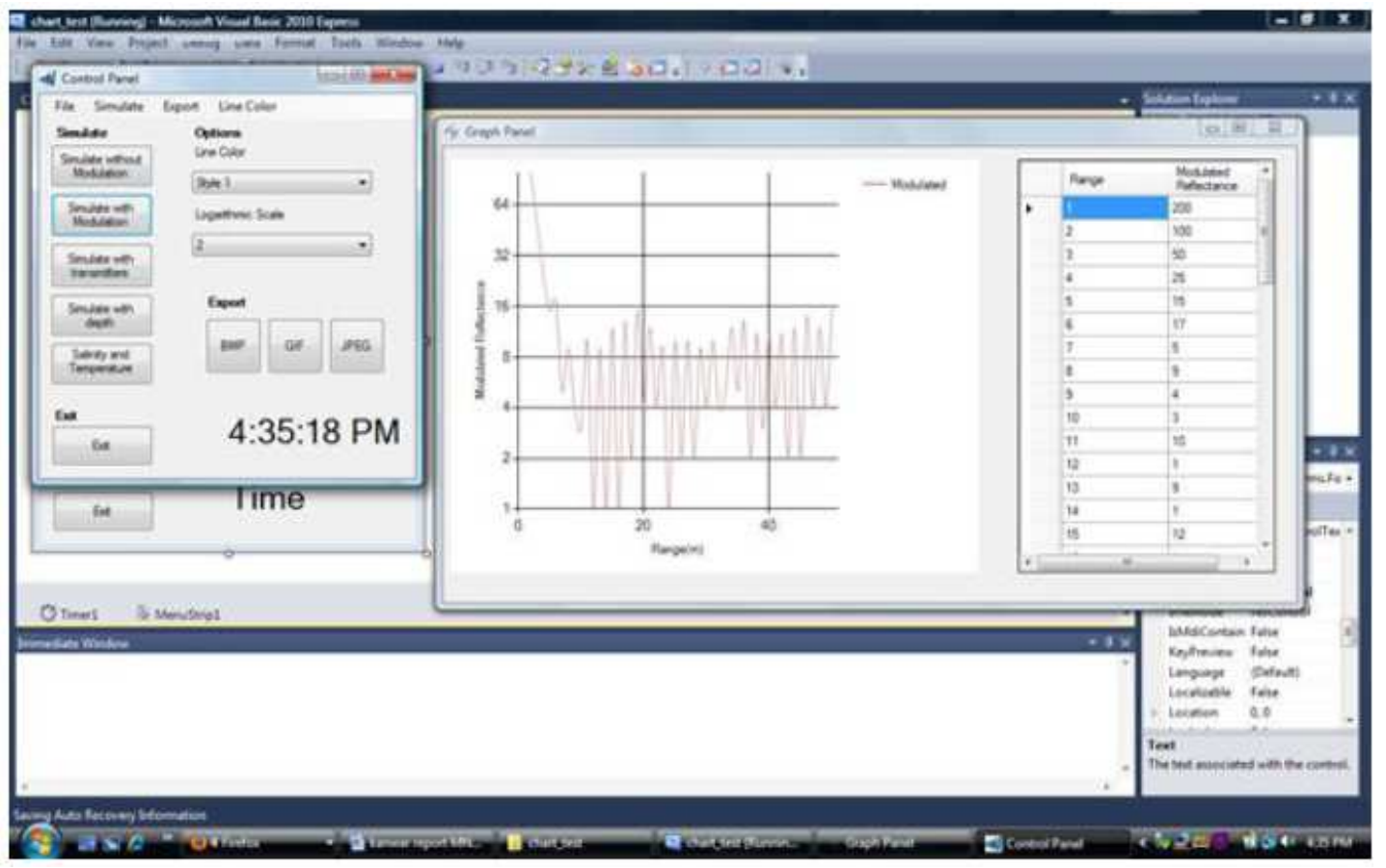

Fig. 5. Screenshot showing simulation without modulation 


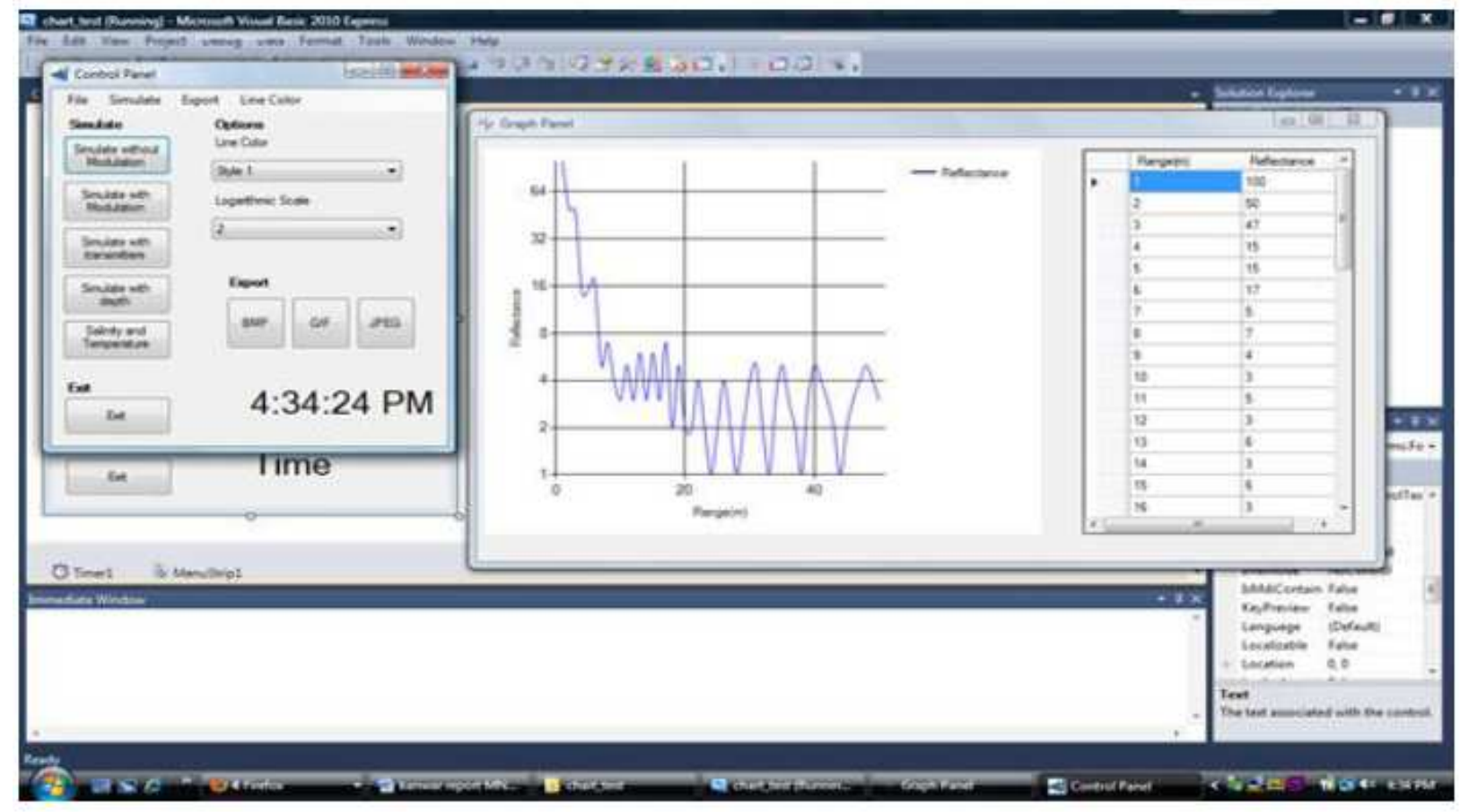

Fig. 6. Screenshot showing simulation with modulation (Reflected energy Vs. distance)

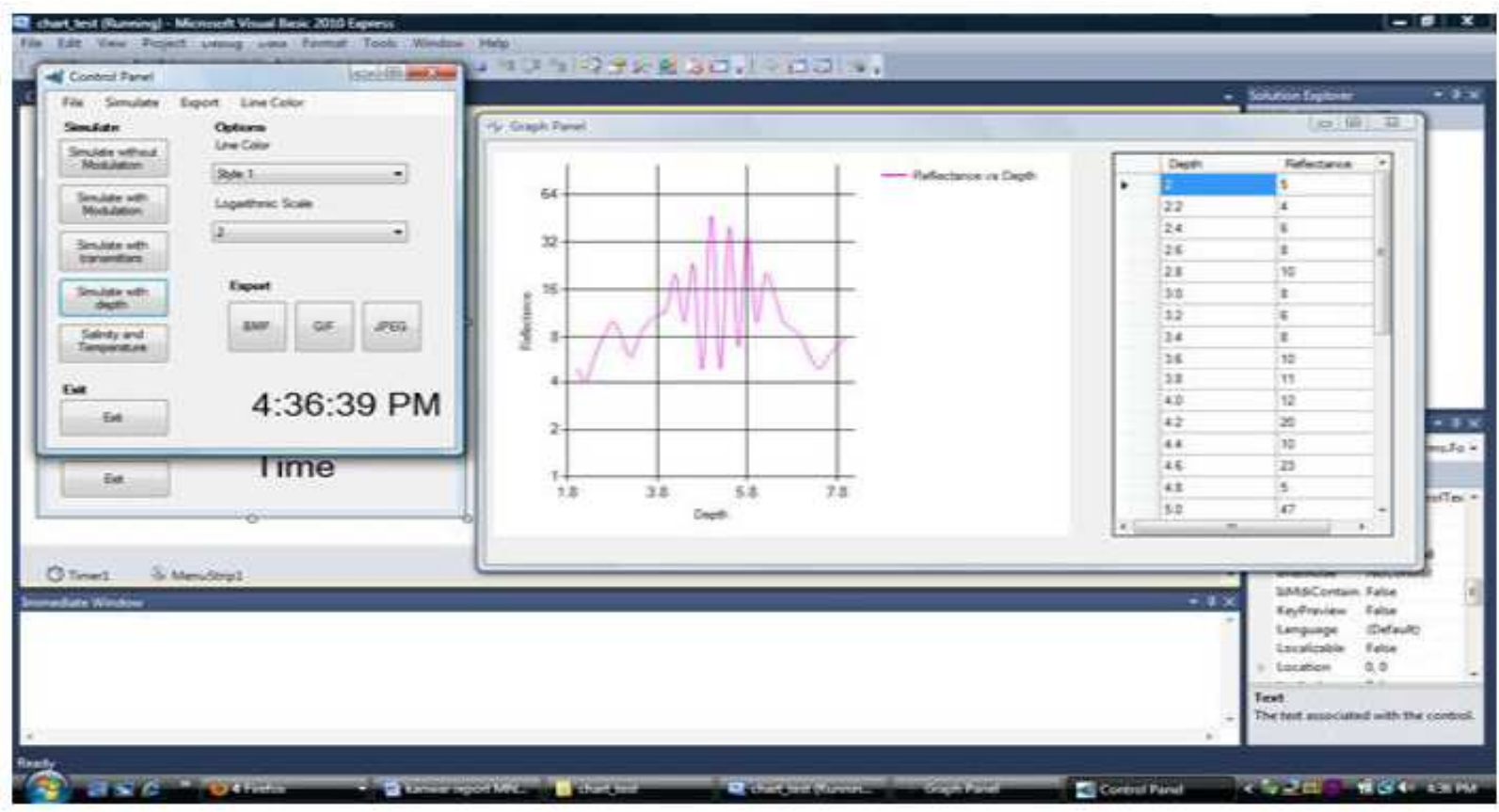

Fig. 7. Screenshot showing simulation with depth (Reflected energy versus receiver depth) 
K. Gilhotra and N.P. Mahalik / American Journal of Engineering and Applied Sciences 7 (3): 327-337, 2014

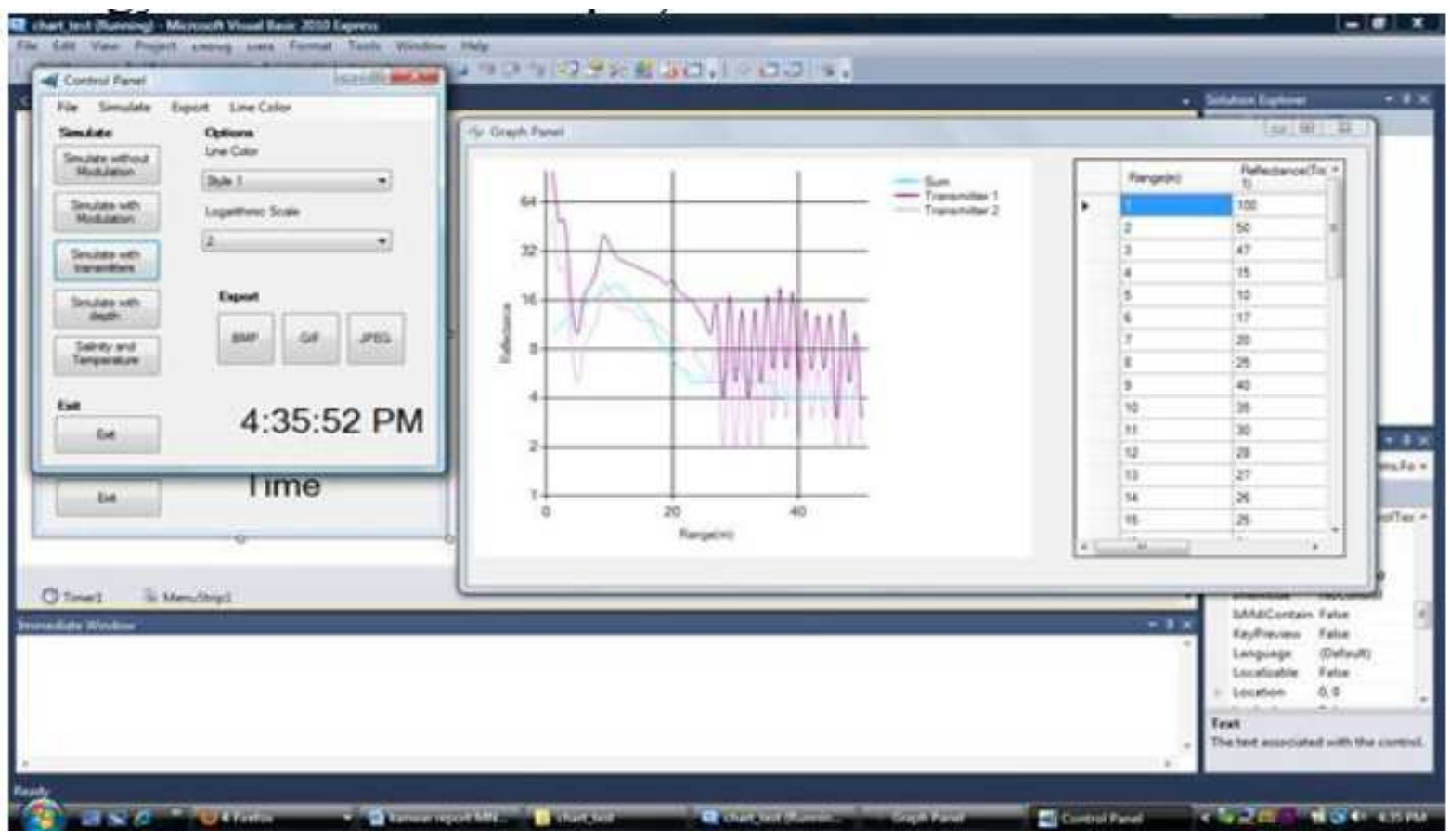

Fig. 8. Screenshot showing simulation with transmitters in array (Reflected energy with receiver array)

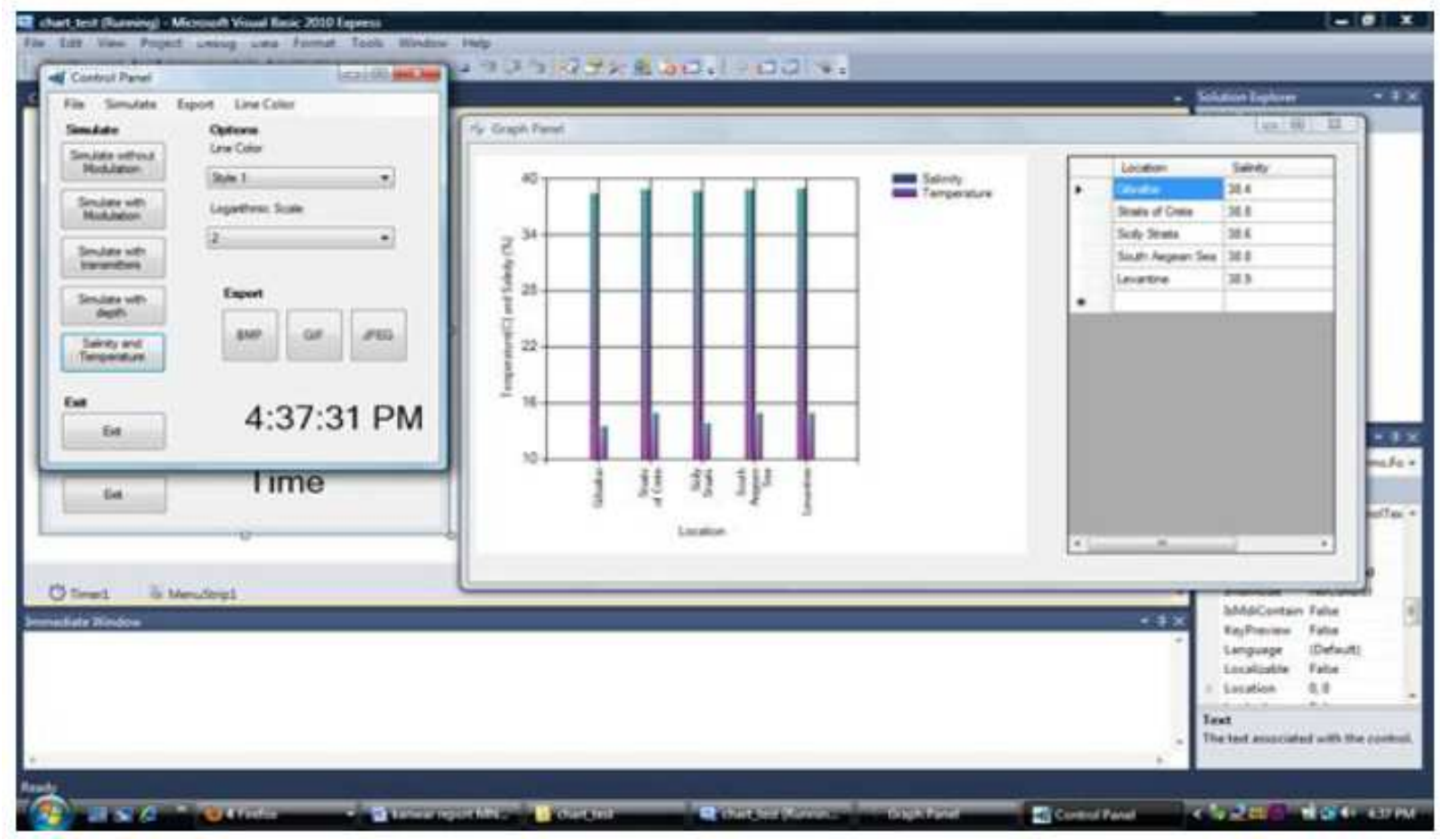

Fig. 9. Screenshot showing simulation of temperature and salinity values 
Figure 6 shows the screenshot of simulated radiation pattern with modulation. Figure 7 shows the screenshot of the software running the simulation with respect to the depth of water. Radiation pattern varies with depth of the ocean. Accordingly, the sensor can be positioned. Figure 8 shows the screenshot of the software running the simulation of radiation pattern when the transmitters are used in array. Note that transmitter array play vital role in underwater communication in order to get rid of multipath problems. Figure 9 shows the screenshot of the software running the simulation of temperature and salinity as variable. Radiation pattern varies with the variation of the salinity and temperature. In order to get the simulated results the designers has to input the parameters and constants such as the speed of sound in water, the receiver depth, the absorption loss, the frequency of the career in case of modulation and so on.

\section{CONCLUSION}

In this study a simulation too was designed to analyze, test and validate the radiation pattern of the Frequency Hopping Spread Spectrum (FHSS) signal intended for underwater sensor network design purposes. We have developed a functional software platform capable of analyzing the radiation pattern effectively. The validation test of the software performed using a given set of data and the results produced by the functional platform is seen to be effective. We tested simulation of radiation pattern (i) without modulation, (ii) with FHSS modulation, (iii) with sensors in array, (iv) with depth of water and (v) with salinity and temperature as variables. The simulation platform has prospective in terms of applications. Now that, we are able to simulate radiation patterns of the underwater sensors in all these various variable conditions depending upon the range, depth, temperature and salinity of water, this software can be expanded to calculate the positioning of the sensors for accurate communication and similarly once this can be incorporated in the software we can further expand the software to formulate the virtual underwater sensor networks for any underwater application. This can reduce the overall design time and thus can reduce costs involved in designing and deploying sensor networks.

\section{ACKNOWLEDGMENT}

The researchers acknowledge Jordan College of Agricultural Sciences and Technology and California
State University Fresno for providing release and assigned time for reviewing research in this area, collecting data and preparing the manuscript. The authors also acknowledge Abhijit Suprem, Smittcamp Scholar, Lyles College of Engineering, California State University, Fresno for helping in developing the aesthetic front end of the simulation platform.

\section{REFERENCES}

Akyildiz, I.F., D. Pompili and T. Melodia, 2005. Underwater acoustic sensor networks: Research challenge. Ad Hoc Netw., 3: 257-279. DOI: 10.1016/j.adhoc.2005.01.004

Bock, C., 2006. SysML and UML 2 support for activity modeling. Syst. Eng., 9: 160-186. DOI: 10.1002/sys.20046

Bouzoualegh, A., T. Val, E. Campo and F. Peyrard, 2005. Modeling and simulation of underwater acoustics communication based on Stateflow and Simulink models. Proceedings of the 3rd International Conference on Sciences of Electronic, Technologies of Information and Telecommunications, Mar. 27-31, SETIT, Tunisia.

Chen, B. and D. Pompili, 2014. A QoS-aware underwater optimization framework for intervehicle communication using acoustic directional transducers. IEEE Trans. Wireless Commun., 13: 2490-2504.

DOI: 10.1109/TWC.2014.031914.131203

Davis, A.M., 1995. 201 Principles of Software Development. 1st Edn., McGraw Hill.

Fourie, A. and D. Nitch, 2000. SuperNEC: Antenna and indoor-propagation simulation program. Antennas Propagat. Magazine IEEE, 42: 31-48. DOI: $10.1109 / 74.848946$

Jiang, Z., 2008. Underwater acoustic networks-issues and solutions. Int. J. Intell. Control Syst., 13: 152161.

Lee, S., C. Kim and K. Kim, 2011. Path planning of a mobile beacon for localization in underwater sensor networks. Proceedings of the IFIP 9th International Conference on Embedded and Ubiquitous Computing, IEEE Computer Society, Oct, 26-24, IEEE Xplore Press, Melbourne, pp: 424-427. DOI: 10.1109/EUC.2011.56

Leyton, M., 2006. Interoperability and objects. Int. J. Product Lifecycle Manage., 1: 98-128. 
Liu, L.M., 2013. A new software development methodology for clinical trial systems. Adv. Software Eng. DOI: 10.1155/2013/796505

Mahalik, N.P. and K. Kim, 2008. A prototype for hardware-in-the-loop simulation of distributed control architecture. IEEE Trans. Syst. Man Cybernet., 38: 189-200. DOI: 10.1109/TSMCC.2007.913891

Mishra, S., 2010. Design and development of management and simulation tool for Distributed Control System (DCS). North Odisha University, India.

Mishra, S., N. Mahalik and K. Kim, 2011. Distributed control systems tools. Proceedings of the Institution of Electrical and Electronics Engineers (IEEE) International Conference on Process Automation, Control and Computing, (ACC' 11), Coimbatore, India.

Nowsheen, N., C. Benson and M. Frater, 2010. Design of a high frequency FPGA acoustic modem for underwater communication. Proceedings of the OCEANS IEEE, May, 24-27, IEEE Xplore Press, Sydney, $\quad$ pp: $1-6 . \quad$ DOI: 10.1109/OCEANSSYD.2010.5603819
Partan, J., J. Kurose and B.N. Levine, 2006. A survey of practical issues in underwater networks. Mobile Comput. Commun. Rev., 11: 23-33. DOI: 10.1145/1347364.1347372

Pompili, D., T. Melodia and I.F. Akyildiz, 2009. A CDMA-based medium access control for underwater acoustic sensor networks. IEEE Trans. Wireless Commun., 8: 1899-1909. DOI: 10.1109/TWC.2009.080195

Radziejewski, I.R., 1990. An investigation of the underwater acoustic communication. MS Thesis, Simon Fraser University, Canada.

Yonghun and K.S. Kim, 2014. Ray tracing assisted distance estimation for underwater acoustic sensor networks. Proceedings of the 3rd Communication and Sensor Network Workshop, (SNW' 14), School of Information and Communication, Gwangju Institute of Sciences and Technology, Gwangju, S. Korea. 